\title{
DISCONTINUITIES IN VELOCITY GRADIENTS AND TEMPERATURE IN THE STOKES' FIRST PROBLEM WITH NONCLASSICAL HEAT CONDUCTION
}

\author{
BY \\ P. PURI AND P. K. KYTHE \\ Department of Mathematics, University of New Orleans, New Orleans, LA
}

\begin{abstract}
The nonclassical heat conduction equation based on the MCF model is used to study the discontinuities in velocity gradients and temperature in fluid flows induced by impulsive or sudden heating of a plate. The influence of the thermal relaxation time in the temperature and velocity fields is investigated.
\end{abstract}

1. Introduction. Fourier's law of heat conduction is used to derive the basic equation in classical unsteady heat transfer problems. This results in a parabolic equation for the temperature field and an infinite speed of heat propagation, thus violating the principle of causality. Ackerman et al [1] established the second sound in solid helium, which gave a finite speed of propagation of thermal waves. Chester [2], Kaliski [3], Lord and Shulman [4], Green and Lindsay [5], and others have developed equations of thermoelasticity, which permit finite speed of thermoelastic propagation. However, no significant progress has been made in developing solutions for corresponding problems in fluid mechanics. Although Lindsay and Straughan [6] have investigated acceleration waves and second sound in a perfect fluid, yet no study has been conducted concerning the existence and propagation of discontinuities in corresponding viscous flows. McTaggart and Lindsay [7] used a non-Fourier heat flux law and analyzed the effect of modified heat conduction equations in the Bénard problem. They have shown that there is a major difference in the results of the Bénard problem when nonclassical effects are taken into account. This is due to the "major role played by the time constant of the MaxwellCattaneo theory". Puri and Kythe [8] have studied a simple unsteady flow problem which deals with the nonclassical heat conduction effects and structure of waves in the Stokes' second problem. A detailed history of the development of nonclassical generalizations of Fourier's law is given in Joseph and Preziosi [9]. They state that the Cattaneo equation is the most obvious and simplest generalization of Fourier's law that gives rise to finite speeds of propagation.

In order to understand fully the effects of the hyperbolic heat conduction equation on fluid flows, we shall investigate the conditions under which discontinuities arise in flows

Received June 17, 1994.

1991 Mathematics Subject Classification. Primary 80A20.

(C) 1997 Brown University 
induced by impulsive or sudden heating of a plate. It is expected that discontinuities will exist in the velocity gradients and temperature. The corresponding thermoelastic problems have been studied by several authors, some of the principal ones being Lord and Shulman [4], and Norwood and Warren [10].

In the so-called MCF [Maxwell-Cattaneo-Fox] model as developed in McTaggart and Lindsay [7], the nonclassical constitutive equation for the heat-flux vector $\mathbf{q}$ is given by the Maxwell-Cattaneo equation

$$
\tau\left(\dot{q}_{i}-\omega_{i j} q_{j}\right)=-q_{i}-\kappa \theta_{, i}
$$

where $\omega_{i j}$ is the vorticity, $\kappa$ the thermal conductivity, $\theta$ the temperature, and $\tau$ the thermal relaxation time. If $\omega_{i j}=0$, Eq. (1) reduces to that of the Cattaneo model, and for $\tau=0$ it becomes Fourier's law (see Joseph and Preziosi [9]). While there are other good models to choose from, the Cattaneo law, as stated in Joseph and Preziosi [9], has many desirable properties, e.g., the steady heat flow may be induced by temperature gradients and gives rise to finite speeds of propagation.

The dimensionless thermal relaxation time, defined as $\lambda=C P$, where $C$ and $P$ are the Cattaneo and the Prandtl numbers respectively, exhibits a definite influence in the existence and speed of propagation of discontinuities in velocity gradients and temperature in fluid flows induced by impulsive or sudden heating of the plate. The number $\lambda$ also appears in generalized thermoelasticity (see Puri [11] where it is defined as $m$ ) and is shown to be of order $10^{-2}$. Again as noted in McTaggart and Lindsay [7], the Cattaneo number $C$ may not be small in astrophysical applications. For example, $C$ is of order $10^{-2}$ in a low temperature hydrogen gas. However, the thermal relaxation time $\lambda$ does not appreciably change the magnitude of the temperature and velocity fields.

The mathematical formulation of the problem and the solutions in the Laplace transform domain and their inversion are contained in $\S 2$. Small-time solutions and propagation of discontinuities in temperature and velocity gradients are investigated in $\S 3$. The results are discussed in $\S 4$, and figures for temperature and velocity profiles are presented there.

2. Mathematical analysis. We will consider the Stokes' first problem (see, e.g., Schlichting [12]). Let a viscous incompressible fluid rest adjacent to a vertical flat plate in the $(y, z)$-plane and occupy the space $x>0$, with the $z$-axis in the vertical direction. The flow is induced by impulsive or sudden heating of the plate, or by time-dependent motion of the plate, or both. The plate initially at rest and at constant temperature $\theta_{\infty}$ (the free stream temperature) is moved with a velocity $U_{0} g(t)$ vertically in its own plane in the direction of the $z$-axis, and its temperature is subjected to heating of the form $\left(\theta_{\omega}-\theta_{\infty}\right) f(t)$, where $\theta_{w}\left(\neq \theta_{\infty}\right)$ is some constant.

The basic equations of continuity, momentum, and energy, governing such a flow, subject to the Boussinesq approximation, are

$$
\begin{gathered}
v_{i, i}=0 \\
\rho \dot{v}_{i}=-p_{. i}+\mu \nabla^{2} v_{i}-\rho\left[1-\alpha\left(\theta-\theta_{\infty}\right)\right] g \delta_{i 3}+t_{k i, k} \\
\rho \dot{\varepsilon}=-q_{i, i}+t_{i k} d_{i k}
\end{gathered}
$$


where the vector $\mathbf{v}=(0,0, u)$ represents the velocity, $\rho$ the density, $\mu$ the dynamic viscosity, $p$ the pressure, $\varepsilon$ the specific internal energy, $\alpha$ the coefficient of thermal expansion, $g$ the acceleration due to gravity, $t_{i k}$ the non-Newtonian stress tensor, and $d_{i k}$ the strain tensor. Taking into account the geometry of the problem which results in the disappearance of the dissipative terms and noting that $t_{i k} \equiv 0$ for the MCF model (see McTaggart and Lindsay [7]), Eqs. (2)-(3) reduce to the following equation of motion:

$$
u_{t}=\nu u_{x x}+g \alpha\left(\theta-\theta_{\infty}\right)
$$

Equation (1), after substitution into (4), gives

$$
\rho c_{p} \dot{\theta}=-q_{i, i}
$$

since $\varepsilon=c_{p} \theta$ for the MCF model. If we drop the nonlinear terms $\tau \omega_{i j} q_{j}$ in (1) because $\tau$ and $\omega_{i j}$ are small quantities, we get

$$
\tau \dot{q}_{i, i}=-q_{i, i}-\kappa \theta_{, i i}
$$

Eliminating $q_{i, i}$ between (6) and (7) we find that

$$
-\rho c_{p} \tau \ddot{\theta}=\rho c_{p} \dot{\theta}-\kappa \theta_{, i i}
$$

which in one-dimensional form, after dropping the convective terms (because these terms become automatically zero), leads to

$$
\tau \theta_{t t}+\theta_{t}=\frac{\kappa}{\rho c_{p}} \theta_{x x}
$$

Note that the term $\tau \theta_{t t}$ in (8) is necessary to ensure finite speed of propagation. We shall use the nondimensional quantities

$$
\begin{gathered}
x=\frac{\nu}{U_{0}} x^{\prime}, \quad u=U_{0} u^{\prime}, \quad t=\frac{\nu}{U_{0}^{2}} t^{\prime}, \quad \frac{\theta-\theta_{\infty}}{\theta_{w}-\theta_{\infty}}=\theta^{\prime} \\
G=\frac{\nu g \alpha\left(\theta_{w}-\theta_{\infty}\right)}{U_{0}^{3}}, \quad P=\frac{\nu \rho c_{p}}{\kappa}, \quad C=\frac{\tau \kappa U_{0}^{2}}{\nu^{2} \rho c_{p}}, \quad \lambda=\frac{\tau U_{0}^{2}}{\nu}=C P,
\end{gathered}
$$

where $G$ is the Grashof number. Then the governing equations (5) and (8) for the flow and heat conduction, after suppressing the primes, become

$$
\begin{gathered}
u_{t}=u_{x x}+G \theta, \\
\lambda P \theta_{t t}+P \theta_{t}=\theta_{x x} .
\end{gathered}
$$

The boundary conditions are

$$
\theta(0, t)=f(t), \quad u(0, t)=g(t), \quad \theta(\infty, t)=0=u(\infty, t)
$$


and the initial conditions are

$$
u(x, 0)=0=\theta(x, 0)=\theta_{t}(x, 0) .
$$

By applying the Laplace transform to the system (10)-(13), the solution in the transform domain is given by

$$
\begin{gathered}
\bar{\theta}(x, s)=\bar{f}(s) e^{-m x} \\
\bar{u}(x, s)=\left[\bar{g}(s)+\frac{G \bar{f}(s)}{m^{2}-s}\right] e^{-x \sqrt{s}}-\frac{G \bar{f}(s)}{m^{2}-s} e^{-m x},
\end{gathered}
$$

where a bar over a quantity denotes its Laplace transform with $s$ as the transform variable, and

$$
m=\sqrt{\lambda P s^{2}+P s} .
$$

For $\bar{f}(s)=1=\bar{g}(s)$, the solutions for $\theta$ and $u=u_{1}-u_{2}$ for $P \neq 1$ are obtained by using the tables of inverse Laplace transforms, e.g., [13]:

$$
\begin{gathered}
\theta(x, t)=H(t-b) a b B(x, t)+\delta(t-b) e^{-a t}, \\
u_{1}(x, t)=H(t)\left[\frac{x}{2 \sqrt{\pi t^{3}}} e^{-x^{2} /(4 t)}-\frac{G}{1-P}\left[\operatorname{erfc}\left(\frac{x}{2 \sqrt{t}}\right)-\frac{1}{2} e^{c t}\{F(x, t, \sqrt{c})\right.\right. \\
+F(x, t,-\sqrt{c}\}]], \\
u_{2}(x, t)=H(t-b) \frac{G}{1-P}\left[e^{c t}\left\{e^{-(c+a) b}+a b \int_{b}^{t} e^{-c \zeta} B(b, \zeta) d \zeta\right\}\right. \\
\left.-\left\{e^{-a b}+a b \int_{b}^{t} B(b, \zeta) d \zeta\right\}\right],
\end{gathered}
$$

where $H(t)$ is the Heaviside step function, $\delta(t)$ the Dirac delta function,

$$
\begin{gathered}
B(x, z)=e^{-a z} \frac{I_{1}\left(a \sqrt{z^{2}-b^{2}}\right)}{\sqrt{z^{2}-b^{2}}} \\
F(x, t, \sqrt{c})=e^{x \sqrt{c}} \operatorname{erfc}\left(\frac{x}{2 \sqrt{t}}+\sqrt{c t}\right),
\end{gathered}
$$

$a=1 /(2 \lambda), b=x \sqrt{\lambda P}$, and $c=(1-P) /(\lambda P)$. For $P=1$, the solution for $u=u_{1}-u_{2}$ in this case is given by

$$
\begin{gathered}
u_{1}=H(t)\left[\frac{x}{2 \sqrt{\pi t^{3}}} e^{-x^{2} /(4 t)}+\frac{G}{\lambda}\left\{\left(t+x^{2} / 2\right) \operatorname{erfc}(x /(2 \sqrt{t}))-\frac{x \sqrt{t}}{\sqrt{\pi}} e^{-x^{2} /(4 t)}\right\}\right] \\
u_{2}=H(t-x \sqrt{\lambda}) \frac{G}{\lambda}\left[t-x \sqrt{\lambda}+\frac{x}{2 \sqrt{\lambda}} \int_{x \sqrt{\lambda}}^{t}(t-\zeta) B(x \sqrt{\lambda}, \zeta) d \zeta\right]
\end{gathered}
$$


For $\bar{f}(s)=1 / s=\bar{g}(s)$, the solutions for $\theta$ and $u=u_{1}-u_{2}$ for $P \neq 1$ are given by

$$
\begin{gathered}
\theta(x, t)=H(t-b)\left[e^{-a b}+a b \int_{b}^{t} B(b, \zeta) d \zeta\right] \\
u_{1}(x, t)=H(t)\left[\operatorname{erfc}(x /(2 \sqrt{t}))+\frac{G \lambda P}{(1-P)^{2}}\left\{\frac{1}{2} e^{c t}(F(x, t, \sqrt{c})+F(x, t,-\sqrt{c}))\right.\right. \\
\left.-\operatorname{erfc}(x /(2 \sqrt{t}))\}-\frac{G}{1-P}\left\{\left(t+x^{2} / 2\right) \operatorname{erfc}(x /(2 \sqrt{t}))-\frac{x \sqrt{t}}{\sqrt{\pi}} e^{-x^{2} /(4 t)}\right\}\right], \\
u_{2}(x, t)=H(t-b)\left[\frac { G \lambda P } { ( 1 - P ) ^ { 2 } } \left\{e^{c t}\left(e^{-(c+a) b}+a b \int_{b}^{t} e^{-c \zeta} B(b, \zeta) d \zeta\right)\right.\right. \\
\left.-\frac{G}{1-P}\left\{(t-b) e^{-a b}+a b \int_{b}^{t}(t-\zeta) B(b, \zeta) d \zeta\right\}\right]
\end{gathered}
$$

For $P=1$, the solution $u=u_{1}-u_{2}$ is given by

$$
\begin{gathered}
u_{1}=H(t)\left[\operatorname{erfc}(x /(2 \sqrt{t}))+\frac{G}{\lambda} \int_{x \sqrt{\lambda}}^{t}\left\{\left(\zeta+z^{2} / 2\right) B(x \sqrt{\lambda}, \zeta)-\frac{x \sqrt{t}}{\sqrt{\pi}} e^{-x^{2} /(4 \zeta)}\right\} d \zeta\right] \\
u_{2}=\frac{1}{2} H(t-x \sqrt{\lambda})\left[\int_{x / \sqrt{\lambda}}^{x}(t-\zeta)^{2} B(x \sqrt{\lambda}, \zeta) d \zeta+(t-x \sqrt{\lambda})^{2}\right]
\end{gathered}
$$

Note that the solutions for other choices of the boundary conditions, like $f(t)=H(t), g(t)$ $=0$, or $f(t)=0, g(t)=H(t)$, can easily be derived from (15).

3. Small-time solutions and propagation of discontinuities. The forcing function $g(t)$ and the part of the velocity given by $u_{1}$ do not introduce any moving discontinuities in the velocity gradients and temperature. We shall, therefore, take $g(t)=0$ in subsequent discussion. Further, there is no need to derive the small time solutions for $u_{1}$ because the exact solutions (18) and (25) are already obtained in terms of well-known functions. The analysis for determining propagation of discontinuities will be carried out in the transform domain. The methodology for this analysis is given in Boley [14], and it was used by Boley and Tolins [15] to study the discontinuities in temperature and stress in coupled thermoelastic half-space. Let $\mathcal{S}\left[f\left(x_{0}\right)\right]$ denote the discontinuity of a function $f(x)$ at a point $x_{0}$, i.e.,

$$
\mathcal{S}\left[f\left(x_{0}\right)\right]=f\left(x_{0}+0\right)-f\left(x_{0}-0\right),
$$

where both $f\left(x_{0}+0\right)$ and $f\left(x_{0}-0\right)$ exist. If

$$
F(x, t)=\frac{1}{2 \pi i} \int_{c-i \infty}^{c+i \infty} \bar{F}(x, s) e^{s t} d t,
$$


where $c$ is a constant chosen in the standard manner of the Laplace inverse transform and $F$ is a piecewise-continuous function of finite exponential order, then the discontinuities are given by the following criterion: Let $\bar{F}(x, s)=\bar{\phi}(x, s) e^{-s \gamma x}$, where $\gamma$ is a real constant. Then, if

$$
\lim _{s \rightarrow \infty} s^{m}\left[\bar{\phi}(x, s)-\frac{K}{s^{n}}\right]=0, \quad n \geq 0, m \geq 1
$$

then

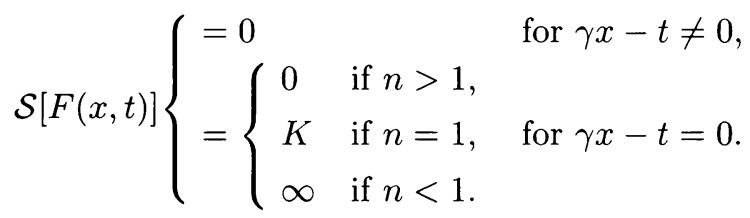

Expansion for large $s$ : When $\bar{f}(s)=1$,

$$
\begin{gathered}
\bar{\theta}(x, s)=e^{-x \sqrt{P /(4 \lambda)}-s x \sqrt{\lambda P}}\left[1+\frac{x}{8 \lambda} \sqrt{\frac{P}{\lambda}} \frac{1}{s}+\cdots\right], \\
\bar{u}_{2}(x, s)=\frac{G}{\lambda P s^{2}} e^{-x \sqrt{P /(4 \lambda)}-s x \sqrt{\lambda P}}\left[1+\left(\frac{1-P}{\lambda P}+\frac{x}{8 \lambda} \sqrt{\frac{P}{\lambda}}\right) \frac{1}{s}+\cdots\right], \\
\frac{\partial \bar{u}_{2}(x, s)}{\partial x}=\frac{G}{\sqrt{\lambda P}} e^{-x \sqrt{P /(4 \lambda)}-s x \sqrt{\lambda P}} \frac{1}{s}\left[1+\left(\frac{1-P}{\lambda P}+\frac{x}{8 \lambda} \sqrt{\frac{P}{\lambda}}+\frac{1}{2 \lambda}\right) \frac{1}{s}+\cdots\right], \\
\frac{\partial u_{2}(x, t)}{\partial t}=s \bar{u}_{2}(x, s) .
\end{gathered}
$$

Inverting (33)-(34), the small-time solutions for $\theta$ and $u_{2}$ are

$$
\begin{aligned}
\theta(x, t)=e^{-x \sqrt{P /(4 \lambda)}}[\delta(t-x \sqrt{\lambda P}) & \left.+\frac{x}{8 \lambda} \sqrt{\frac{P}{\lambda}} H(t-x \sqrt{\lambda P})+\cdots\right], \\
u_{2}(x, t)=H(t-x \sqrt{\lambda P}) \frac{G}{\lambda P} e^{-x \sqrt{P /(4 \lambda)}}[t & -x \sqrt{\lambda P} \\
& \left.+\left(\frac{1-P}{\lambda P}+\frac{x \sqrt{P}}{8 \lambda \sqrt{\lambda}}\right) \frac{(t-x \sqrt{\lambda p})^{2}}{2}+\cdots\right] .
\end{aligned}
$$

By applying the criterion (32) to (33) it is seen that discontinuity in $\theta(x, t)$ at the wave-front at $x=t / \sqrt{\lambda P}$ is unbounded, but this discontinuity consists of two parts, one being an impulse of magnitude $-e^{-x \sqrt{P /(4 \lambda)}}$, and the other a jump of magnitude $\frac{x}{8 \lambda} \sqrt{\frac{P}{\lambda}} e^{-x \sqrt{P /(4 \lambda)}}$. Again, we find from $(34)-(36)$ that $u_{2}(x, t)$ is continuous everywhere, but $\partial u_{2} / \partial x$ and $\partial u_{2} / \partial t$ have discontinuities of magnitude $-\frac{G}{\sqrt{\lambda P}} e^{-x \sqrt{P /(4 \lambda)}}$ and $\frac{G}{\lambda P} e^{-x \sqrt{P /(4 \lambda)}}$ respectively at the same wave-front. These discontinuities propagate with speed $1 / \sqrt{\lambda P}$. 
When $\bar{F}(s)=1 / s$, the small-time solutions for $\theta$ and $u_{2}$ are

$$
\begin{gathered}
\theta(x, t)=e^{-x \sqrt{P /(4 \lambda)}}[H(t-x \sqrt{\lambda P})+\cdots], \\
u_{2}(x, t)=H(t-x \sqrt{\lambda P}) \frac{G}{\lambda P} e^{-x \sqrt{P /(4 \lambda)}}\left[\frac{(t-x \sqrt{\lambda P})^{2}}{2}\right. \\
\left.+\left(\frac{1-P}{\lambda P}+\frac{x \sqrt{P}}{8 \lambda \sqrt{\lambda}}\right) \frac{(t-x \sqrt{\lambda P})^{3}}{6}+\cdots\right] .
\end{gathered}
$$

The discontinuity in $\theta(x, t)$ at the wave-front at $x=t / \sqrt{\lambda P}$ is of magnitude $e^{-x \sqrt{P /(4 \lambda)}}$. It is seen that $u_{2}(x, t)$ and its first gradients are continuous at all points, but the second gradients have discontinuities given by $\mathcal{S}\left[\partial^{2} u_{2} / \partial x^{2}\right]=G e^{-x \sqrt{P /(4 \lambda)}}$, and $\mathcal{S}\left[\partial^{2} u_{2} / \partial t^{2}\right]$ $=\frac{G}{\lambda P} e^{-x \sqrt{P /(4 \lambda)}}$ at the same wave-front.

4. Discussion. Table 1 summarizes the results for the magnitudes of discontinuities at the wave-front $x=t / \sqrt{\lambda P}$ in the temperature and the velocity gradients.

TABLE 1

\begin{tabular}{cccccc}
\hline$f(t)$ & $\mathcal{S}[\theta(x, t)]$ & $\mathcal{S}[\partial u / \partial t]$ & $\mathcal{S}\left[\partial^{2} u / \partial t^{2}\right]$ & $\mathcal{S}[\partial u / \partial x]$ & $\mathcal{S}\left[\partial^{2} u / \partial x^{2}\right]$ \\
\hline$\delta(t)$ & $\infty$ & $\frac{G}{\lambda P} e^{-x \sqrt{P /(4 \lambda)}}$ & $\infty$ & $-\frac{G}{\sqrt{\lambda P}} e^{-x \sqrt{P /(4 \lambda)}}$ & $\infty$ \\
$H(t)$ & $e^{-x \sqrt{P /(4 \lambda)}}$ & 0 & $-\frac{G}{\lambda P} e^{-x \sqrt{P /(4 \lambda)}}$ & 0 & $-G e^{-x \sqrt{P /(4 \lambda)}}$ \\
\hline
\end{tabular}

The interesting feature of this model is that discontinuities are present in the temperature and the velocity gradients. Although we have derived the results for both impulsive and sudden heating or motion of the plate, we shall confine our discussion to the more practical case of sudden heating. The results derived for $f(t)=\delta(t)=g(t)$ constitute the Green's function in some sense (with respect to time); they are, therefore, basic results of theoretical importance. It is obvious from Table 1 that the jumps for velocity gradients are very large except for the case of $\partial^{2} u / \partial x^{2}$ for $f(t)=H(t)$.

The discontinuities in temperature disappear very quickly. It is clear from Fig. 1 (see p. 174) that for $t=0.05$ the discontinuity in $\theta$ is very small and temperature is almost zero just ahead of the wave-front. However, numerical computation shows that the discontinuities in velocity gradients start with large magnitudes and propagate for about ten seconds (for the specific values of $\lambda, P$, and $G$ considered here) before they start to disappear. Figure 1 exhibits the temperature profiles for small times. It is clear that the magnitude of discontinuities in temperature at the wave-front (marked by the vertical broken line) decreases with time and practically disappears before $t=0.05$.

The temperature profiles for $\lambda=0$ are given in Fig. 2, and velocity profiles for $\lambda=0$ and small times are given in Fig. 3. They are provided for comparison with our results.

Figures 3 and 4 (see p. 175) show that (i) the effect of $\lambda$ is minimal on velocity profiles, (ii) an increase in $G$ increases the velocity, and (iii) the cooling $(G<0)$ and 


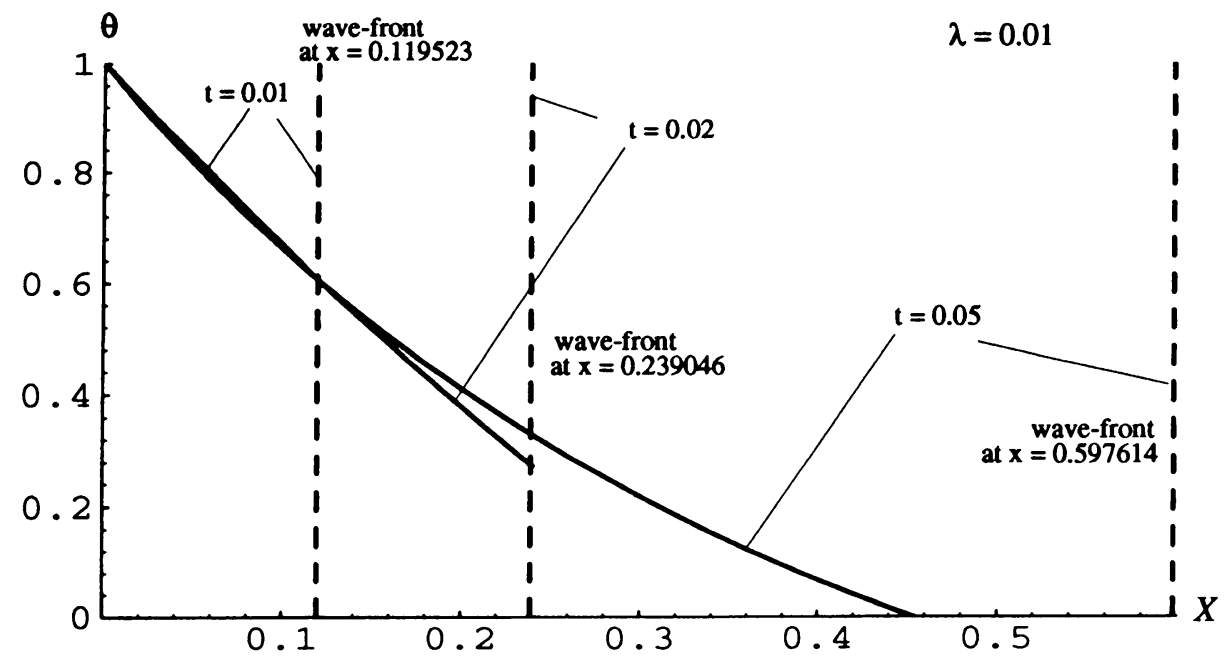

FIG. 1. Graphs of $\theta(x, 0.01), \theta(x, 0.02)$, and $\theta(x, 0.05)$ for $P=$ $0.7, \lambda=0.01$.

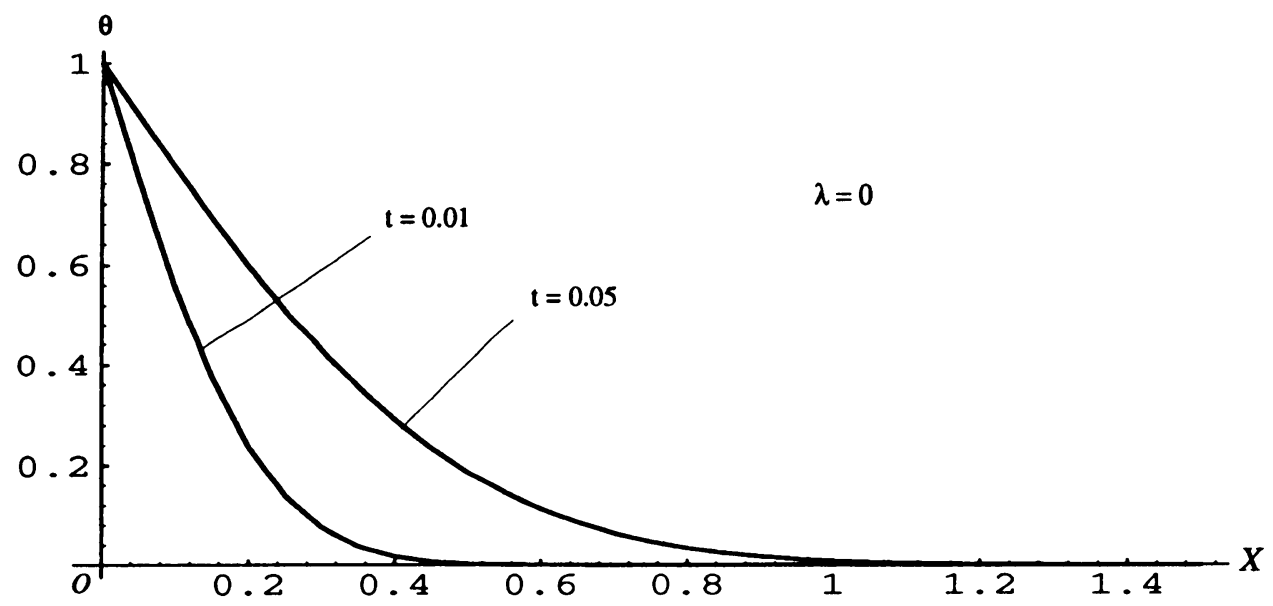

FIG. 2. Temperature when $\lambda=0$, for $P=0.7$, and $t=0.01$ and 0.05 .

heating $(G>0)$ effects become more prominent as time increases. It is also clear from Tables 2 and 3 that an increase in $\lambda$ tends to decrease the velocity for $G>0$ and increase it for $G<0$.

TABLE 2. Velocity at $t=0.05, P=0.7, G=5$.

\begin{tabular}{lccccccc}
\hline$x$ & 0.0 & 0.5 & 0.2 & 0.4 & 0.6 & 0.8 & 1.0 \\
$\lambda=0.01$ & 1.0 & 0.901984 & 0.583310 & 0.239717 & 0.065572 & 0.012171 & 0.001621 \\
$\lambda=0.0$ & 1.0 & 0.902823 & 0.587058 & 0.247933 & 0.075167 & 0.016388 & 0.002600 \\
\hline
\end{tabular}




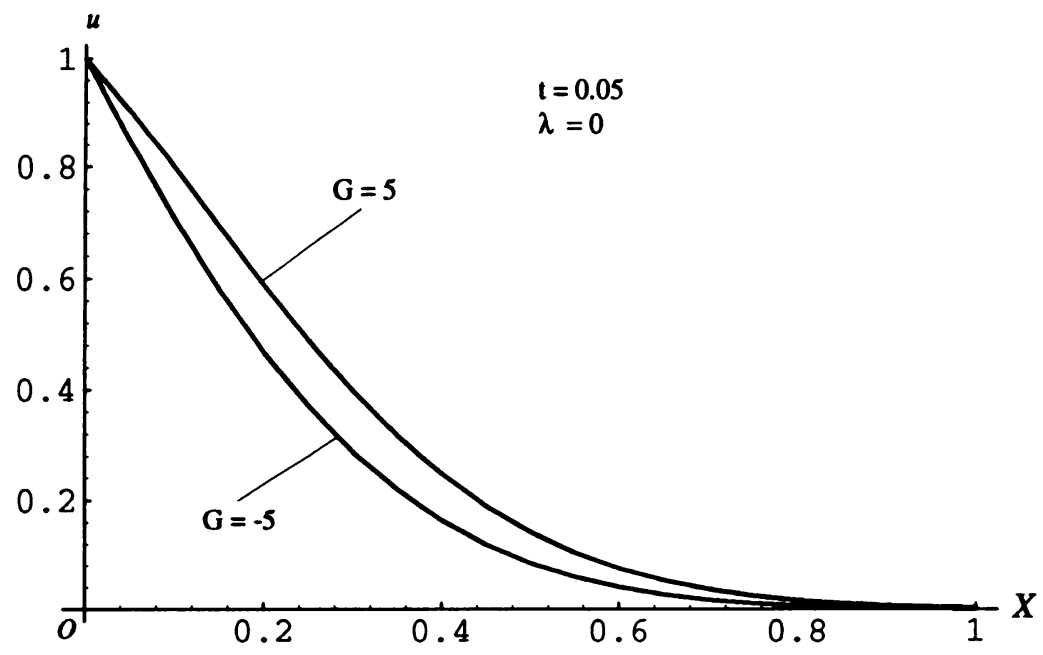

Fig. 3. Velocity when $\lambda=0$, for $P=0.7, G= \pm 5$, and $t=0.05$.

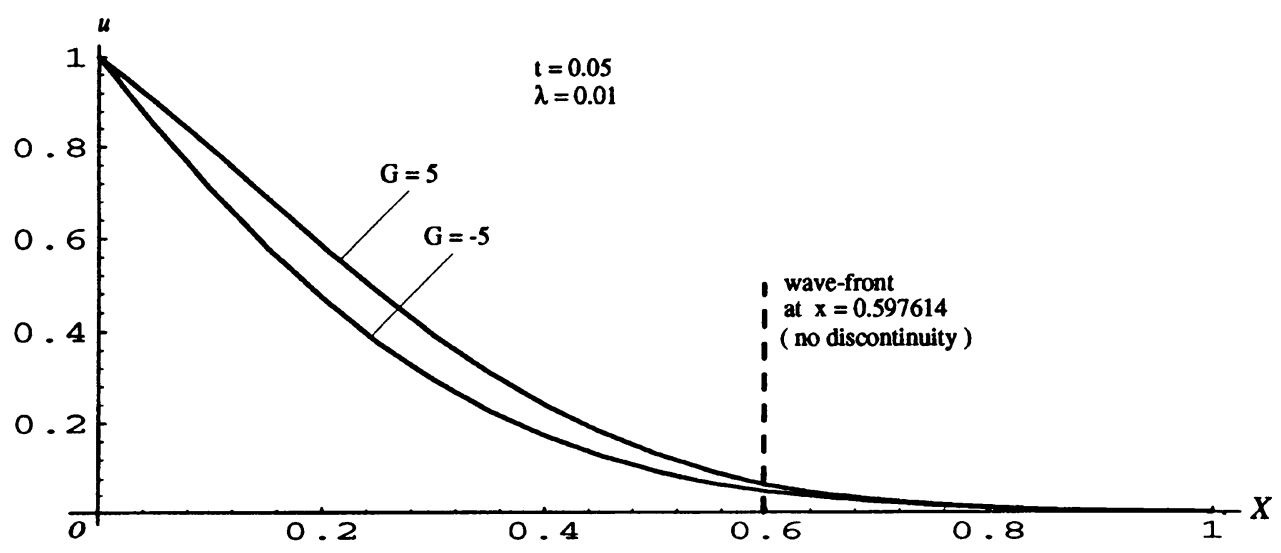

FIG. 4. Velocity when $\lambda=0.01$, for $P=0.7, G= \pm 5$, and $t=0.05$.

TABle 3. Velocity at $t=0.05, P=0.7, G=-5$.

\begin{tabular}{lccccccc}
\hline$x$ & 0.0 & 0.05 & 0.2 & 0.4 & 0.6 & 0.8 & 1.0 \\
$\lambda=0.01$ & 1.0 & 0.846750 & 0.470869 & 0.172087 & 0.049986 & 0.010652 & 0.001509 \\
$\lambda=0.0$ & 1.0 & 0.845911 & 0.467121 & 0.163874 & 0.040392 & 0.006435 & 0.000530 \\
\hline
\end{tabular}

Reciprocal relations of the type mentioned in Boley and Tolins [15] do not exist in the present work.

Acknowledgment. The authors are grateful to the referee for helpful suggestions. 


\section{REFERENCES}

[1] C. C. Ackerman, B. Bertman, H. A. Fairbank, and R. A. Guyer, Second sound in solid helium, Phys. Rev. Lett. 16, 789-791 (1966)

[2] M. Chester, Second sound in solids, Phys. Rev. 131, 2013-2015 (1963)

[3] S. Kaliski, Wave equations in thermoelasticity, Bull. Acad. Polon. Sci., Ser. Sci. Tech. 13, 253-260 (1965)

[4] H. W. Lord and Y. Shulman, A generalized dynamical theory of thermoelasticity, J. Mech. Phys. Solids 15, 299-309 (1967)

[5] A. E. Green and K. A. Lindsay, Thermoelasticity, J. Elast. 2, 1-7 (1972)

[6] K. A. Lindsay and B. Straughan, Acceleration waves and second sound in a perfect fluid, Arch. Rational Mech. Anal. 68, 53-87 (1978)

[7] C. L. McTaggart and K. A. Lindsay, Nonclassical effects in the Bénard problem, SIAM J. Appl. Math. 45, 70-92 (1985)

[8] P. Puri and P. K. Kythe, Nonclassical thermal effects in Stokes' second problem, Acta Mechanica 112, 1-9 (1995)

[9] D. D. Joseph and L. Preziosi, Heat waves, Rev. Modern Phys. 61, 41-73 (1989)

[10] F. R. Norwood and W. E. Warren, Wave propagation in the generalized dynamical theory of thermoelasticity, Quart. J. Mech. Appl. Math. 22, 283-290 (1969)

[11] P. Puri, Plane waves in generalized thermoelasticity, Internat. J. Engrg. Sci. 11, 735-744 (1973); 13, 339-340 (1975)

[12] H. Schlichting, Boundary Layer Theory, 4th Ed., McGraw-Hill, New York, 1960, p. 72

[13] A. Erdélyi (Ed.), Tables of Laplace Transforms, Vol. 1, Bateman Manuscript Project, McGrawHill, New York, 1954

[14] B. A. Boley, Discontinuities in integral-transform solutions, Quart. Appl. Math. 19, 273-284 (1962)

[15] B. A. Boley and I. S. Tolins, Transient coupled thermoelastic boundary value problems in the half-space, J. Appl. Mech. 29, Trans. ASME 84, 637-646 (1962) 